Article

\title{
The Significance of the Alter miR let-7a and miR-335 Expression Level Regulating the CCR7/CCL19 Axis as Potential Biomarkers of Tumor Progression in NSCLC
}

\author{
Kamila Baran 1,*1D, Jacek Kordiak ${ }^{2}$, Sławomir Jabłoński ${ }^{2}$, Adam Antczak ${ }^{3}$ and Ewa Brzeziańska-Lasota ${ }^{1}$ (D) \\ 1 Department of Biomedicine and Genetics, Medical University of Lodz, 92-213 Lodz, Poland; \\ ewa.brzezianska@umed.lodz.pl \\ 2 Department of Thoracic, General and Oncological Surgery, Medical University of Lodz, 90-549 Lodz, Poland; \\ jacek.kordiak@umed.lodz.pl (J.K.); slawomir.jablonski@umed.lodz.pl (S.J.) \\ 3 Department of General and Oncological Pulmonology, Medical University of Lodz, 90-153 Lodz, Poland; \\ adam.antczak@umed.lodz.pl \\ * Correspondence: kamila.baran@umed.lodz.pl
}

Citation: Baran, K.; Kordiak, J.; Jabłoński, S.; Antczak, A.;

Brzeziańska-Lasota, E. The Significance of the Alter miR let-7a and miR-335 Expression Level Regulating the CCR7/CCL19 Axis as Potential Biomarkers of Tumor Progression in NSCLC. J. Clin. Med. 2022, 11, 655. https://doi.org/ $10.3390 / j \mathrm{~cm} 11030655$

Academic Editor: Ramon Andrade De Mello

Received: 26 November 2021

Accepted: 21 January 2022

Published: 27 January 2022

Publisher's Note: MDPI stays neutral with regard to jurisdictional claims in published maps and institutional affiliations.

Copyright: (c) 2022 by the authors. Licensee MDPI, Basel, Switzerland. This article is an open access article distributed under the terms and conditions of the Creative Commons Attribution (CC BY) license (https:/ / creativecommons.org/licenses/by/ $4.0 /)$.

\begin{abstract}
The chemokine receptor 7/C-C ligand 19 chemokine (CCR7/CCL19) has been implicated in the development and progression of NSCLC. Its expression is regulated by various epigenetic factors including miRNAs. The aim of this study was to assess the expression of CCR7/CCL19 in cancer tissue in relation to that of miRNAs (miR-let-7a, miR-335) as transcriptional regulators. The expression of the tested miRNAs was also evaluated in serum exosomes. Sixty patients $(n=60)$ were enrolled in the study. The total expression of the studied mRNA and miRNAs were evaluated using qPCR. Tumor tissue fragments, macroscopically unchanged adjacent tissue, and serum were used as controls. Higher CCR7 and CCL19 mRNA expression levels were observed in tumor tissue compared to control. According to stages of the disease (AJCC tumor staging), the greatest expression level of the studied genes' mRNA was observed in patients with stage III. In NSCLC patients, lower miR let-7a expression level was observed in tumor tissue compared to serum; however, miR-335 expression level was higher $(p<0.05)$. The expression level of miR-335 positively correlated with tumor size ( $\mathrm{T}$ features according to pTNM staging) and AJCC tumor staging, while miR let-7a had a negative correlation $(p>0.05)$ with liquid biopsy. Significantly greater miR-335 expression level and lower miR let-7a expression level in serum were observed in patients with metastases to lymph nodes. Our findings reveal a significant correlation between the expression levels of the mRNA of the studied genes and miRNAs. Changes in miR-335 and miR let-7a expression levels in the serum exosomes of NSCLC patients in relation to lymph node metastases and tumor stage may serve as a non-invasive molecular biomarker of tumor progression.
\end{abstract}

Keywords: non-small lung cancer; real-time polymerase chain reaction; CCR7; CCL19; miR-335; miR let-7a

\section{Introduction}

Lung cancer is one of the most commonly diagnosed cancers and the leading cause of cancer-related mortality. According to WHO data, 2,206,771 new cases of lung cancer were recorded in 2020, which accounts for $11.4 \%$ of all cancer cases [1]. It was as responsible for the deaths of approximately 1,796,144 people worldwide last year, 27,444 of which were from Poland. The most frequently diagnosed histological type is non-small cell lung cancer (NSCLC), which accounts for $85 \%$ of cases. The high mortality of lung cancer, including NSCLC, is caused mainly by the late diagnosis of patients with advanced-stage cancer. A better understanding of the molecular mechanisms responsible for the development and progression of NSCLC will reveal biomarkers that can be used for diagnosis.

The system of chemokines and their receptors plays an important role in the progression of cancer. The discovery of the CC chemokine receptor 7 (CCR7) in the membrane 
of lung cancer cells suggests that it may be utilized to migrate along the gradient of C-C motif chemokine 19 (CCL19) and chemokine 21 (CCL21) towards the lymph node and subsequently cause lymph node metastasis [2]. Furthermore, CCR7 and its ligands have been implicated in the occurrence and progression of NSCLC $[3,4]$. It was revealed that CCR7 expression is induced by hypoxia, which enhances the migration and invasion of lung cancer cells. Furthermore, CCR7 play a key role in the regulation of cell proliferation, apoptosis, and epithelial-mesenchymal transition (EMT) via the ERK and NF- $k B$ pathway $[3,5,6]$. The recent study revealed that matrine, alkaloid isolated from plants of Saphora species, induces cell apoptosis in NSCLC cell line via targeting CCR7 [7]. Moreover, inhibition of CCR7 expression by metrine enhances effects of anticancer treatment, such as cisplatin, 5-fluorouracil, and paclitaxel in vitro. Thus, CCR7 may serve as a novel therapeutic target for NSCLC. Furthermore, the study performed in murine models of lung cancer demonstrated that local and systemic administration of CCL19 combined with IL-7 is beneficial as potent anti-cancer strategy by stimulation of the immune response [8]. Other research revealed that tumor microenvironment cells, such as fibroblastic stromal cells, produce CCL19, which promote local antitumor T-cell responses and restrain the growth of lung carcinoma [9]. Moreover, Itakura et al. [10] revealed that a high CCL19 expression level was a good prognostic factor in lung adenocarcinoma. Hence, the diagnostic value of the CCR7/CCL19 axis in lung tumorigenesis remains controversial [2,10].

It has been revealed that microRNAs (miRNAs) may regulate the expression of the messenger ribonucleic acid (mRNA) of chemokines or their receptors and play a role in various cellular processes implicated in the development and progression of NSCLC [11,12]. miRNA are single-stranded RNA chains of approximately 22 nucleotides that do not encode proteins. These molecules negatively regulate the post-transcriptional level of target mRNAs by binding to the $3^{\prime}$-untranslated region (UTR), resulting in the degradation of mRNA or translation inhibition [13]. They can exert oncogenic and tumor-suppressive effects on cancer cells influencing tumor microenvironment [14]. Moreover, oncogenic miRNAs may participate in tumor progression by their transfer of exosomes to other regions of the body, where the promotion of metastasis occurs. Dysregulation in miRNAs expression during tumorigenesis may be caused by various mechanisms, including the amplification or deletion of miRNA genes, abnormal transcriptional control of miRNAs, and dysregulated epigenetic changes [15]. An alternation in miRNA expression levels may serve as diagnostic and/or prognostic biomarkers in NSCLC in liquid biopsy.

This research is a continuation and extension of our earlier study, the aim of which was to evaluate changes in the expression level of CCR7 and CCL19 in tumor tissue and of two regulatory miRNAs of these genes (miR let-7a and miR-335) in serum exosomes [16]. A particularly valuable element of this study is the measurement of miRNA expression level in tumor tissue. It also compares the expression profiles of selected miRNAs originating from the exosomes and tumor tissue of NSCLC patients and analyzes the differences in the expression of the chosen miRNAs, CCR7 and CCL19, with regard to selected clinical features of NSCLC patients.

\section{Experimental Section}

\subsection{Subjects}

The study cohort consisted of 60 patients with diagnosed NSCLC, 25 women and 35 men, aged 51 to 81 (mean age $67.13 \pm 7.14$ years). Lung resection (pulmonectomy or lobectomy) in patients was performed at the Department of Thoracic Surgery, General and Oncological Surgery, Military Medical Academy Memorial Teaching Hospital of the Medical University of Lodz-Central Veterans' Hospital, Lodz, Poland during the years 2018-2019. The clinical features of the patients with diagnosed NSCLC are summarized in Table 1. 
Table 1. Demographic characteristics of patients and features of lung cancer.

\begin{tabular}{|c|c|}
\hline Patient Characteristics & Number of Patients (\%) \\
\hline \multicolumn{2}{|l|}{ Sex } \\
\hline Female & $25(41.67 \%)$ \\
\hline Male & $35(58.33 \%)$ \\
\hline \multicolumn{2}{|l|}{ Age } \\
\hline$\leq 65$ & $28(46.67 \%)$ \\
\hline$>65$ & $32(53.33 \%)$ \\
\hline \multicolumn{2}{|l|}{ Smoking status and smoking history } \\
\hline$<40 \mathrm{PYs}^{\mathrm{a}}$ & $26(43.33 \%)$ \\
\hline$\geq 40$ PYs & $26(43.33 \%)$ \\
\hline Non-smoking & $7(11.67 \%)$ \\
\hline Tumor Characteristics & Number of Cases (\%) \\
\hline \multicolumn{2}{|l|}{ Histopathological type of NSCLC } \\
\hline SSC & $28(46.67 \%)$ \\
\hline $\mathrm{AC}$ & $32(53.33 \%)$ \\
\hline \multicolumn{2}{|l|}{$\mathrm{AJCC}^{\mathrm{b}}$ staging system } \\
\hline Stage I & $30(50 \%)$ \\
\hline Stage II & $21(35 \%)$ \\
\hline Stage III & $9(15 \%)$ \\
\hline \multirow{2}{*}{\multicolumn{2}{|c|}{$\begin{array}{l}\text { Metastasis to lymph nodes spread according to the } \\
\text { pTNM }^{c} \text { staging system }\end{array}$}} \\
\hline & \\
\hline No & $39(65 \%)$ \\
\hline $\mathrm{N} 1+\mathrm{N} 2$ & $21(35 \%)$ \\
\hline \multicolumn{2}{|c|}{$\begin{array}{l}\text { N1 }+\mathrm{N} 2 \\
\text { Tumor size according to the pTNM staging system }\end{array}$} \\
\hline $\mathrm{T} 1 \mathrm{a}+\mathrm{T} 1 \mathrm{~b}$ & $21(35 \%)$ \\
\hline $\mathrm{T} 2 \mathrm{a}+\mathrm{T} 2 \mathrm{~b}$ & $29(48.33 \%)$ \\
\hline T3 & $10(16.67 \%)$ \\
\hline
\end{tabular}

\subsection{Tissue and Serum Collection}

Primary tumor tissue fragments from diagnosed NSCLC patients were obtained from the middle of the lesion (approximately $100 \mathrm{mg}$ ). Adjacent macroscopically unchanged tissue (approximately $100 \mathrm{mg}$ taken at a distance of $2 \mathrm{~cm}$ from the primary focus) was used as the control. The resected primary tumors were classified as either squamous cell carcinoma (SCC) or adenocarcinoma (AC). The tissue samples were subjected to a postoperative histopathological evaluation and classified according to the AJCC and TNM staging systems (pTNM) [18]. The histopathological characteristics of the biological materials is shown in Table 1.

Serum from all $(n=60)$ study patients with diagnosed NSCLC were collected before the surgery. Sera from 45 patients (potentially healthy volunteers) were used as controls.

The biological material was secured and prepared according to the protocol described in the previous article [16]. Isolation of total RNA from tissue and exosomes was performed with the same type of isolation kits as before. Qualitative and quantitative assessment of the RNA was performed by spectrophotometry (260/280 nm), using an Eppendorf BioPhotometerTM Plus apparatus (Eppendorf, Hamburg, Germany).

\subsection{Assessment of the Relative Level of Genes and miRNAs Expression (RQ)}

The genes were subjected to reverse transcription (RT) using a High-Capacity cDNA Reverse Transcription Kit (Applied Biosystems, Carlsbad, CA, USA) in a Personal Thermocycler (Eppendorf, Germany). The relative expression level of the study genes was analyzed in a 7900HT Fast Real-Time PCR System (Applied Biosystems, Carlsbad, CA, USA) using TaqMan assay for the genes CCR7 (Hs01013469_m1), CCL19 (Hs00171149_m1), and 
ACTB (Hs99999903_m1), used as an endogenous control. The reverse transcription (RT) for miRNA was carried out using a TaqMan ${ }^{\circledR}$ MicroRNA Reverse Transcription Kit (Applied Biosystems, Carlsbad, CA, USA) according to the manufacturer's protocol. The RT reaction was performed in a Personal Thermocycler (Eppendorf, Germany). The components of reactions and conditions were kept as before.

The relative expression level of the study genes was evaluated by the delta-delta CT method (TaqMan Relative Quantification Assay software, Applied Biosystems). RNA from high quality control standard lung tissue (Human Lung Total RNA, Ambion ${ }^{\circledR}$, Austin, TX, USA) was used as a calibrator.

The relative $R Q$ value of the studied miRNAs was assessed by global normalization. Calibration was performed using commercial RNA (Human Lung Total RNA, Ambion ${ }^{\circledR}$, Austin, TX, USA), and the median CT of those assays (on a per sample basis) was used to evaluate the expression level of the studied miRNAs in tumor tissue and serum. The level of calibrator expression was regarded as $\mathrm{RQ}=1$.

\subsection{Statistical Analysis}

The data was analyzed using the Mann-Whitney U-test and/or the Kruskal-Wallis test depending on the size of the groups. The Spearman rank correlation coefficient was used to measure the direction and strength of the association for individual variables. All analyses were performed using Statistica 13.1 software (StatSoft, Cracow, Poland). For all statistical analyses, the level of statistical significance was adopted at $p<0.05$. RQ values for the study genes/miRNAs were presented as median values.

\section{Results}

\subsection{Relative Expression Levels of the Studied mRNA in Tumor Tissue (NSCLC) vs. Control Lung Tissue}

CCR7 mRNA expression was upregulated $(\mathrm{RQ}>1)$ in two study groups: lung cancer tissue (98\%) and macroscopically unchanged lung tissue (100\%). Also, higher expression levels of CCR7 mRNA were observed in tumor tissue compared to the control tissue (median RQ: 11.268 and 8.747 , respectively), but this difference was not statistically significant ( $p>0.05$, Mann-Whitney U-test) (Figure 1).

CCL19 mRNA expression level was upregulated (RQ $>1$ ) in 53\% of lung cancer tissue samples and in $40 \%$ of macroscopically unchanged controls. A statistically significant difference was observed in the CCL19 mRNA expression level between tumor tissue (NSCLC) and control tissue ( $p=0.019$, Mann-Whitney U-test), with a higher CCL19 mRNA expression level in tumor tissue (median RQ: 1.052 and 0.709, respectively) (Figure 1).
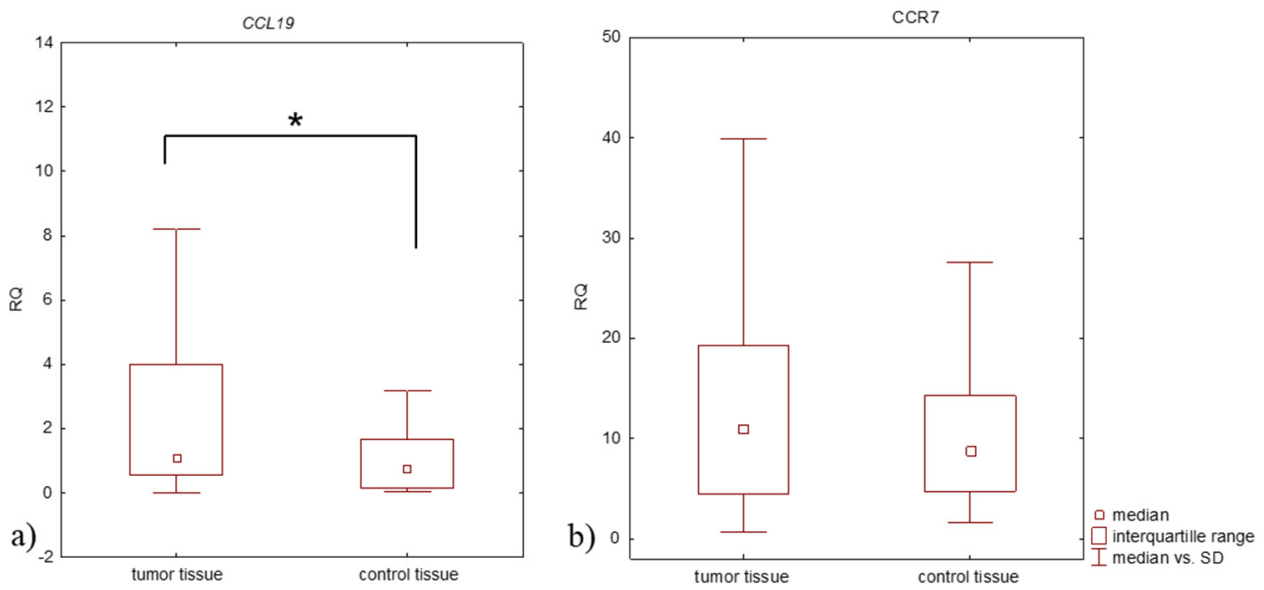

Figure 1. Box plot showing differences in RQ medians for (a) CCL19, (b) CCR7 in tumor and control tissue in patients with NSCLC (Mann-Whitney U-test); ${ }^{*} p<0.05$. 
3.2. Relative Expression Levels of CCR7 and CCL19 mRNA in Tumor Tissue (NSCLC) According to Biological Features and Smoking History of Study Patients

The expression level of CCR7 mRNA in tumor tissue was higher in female patients compared to the male group (median RQ: 13.548 and 10.796, respectively), as well as in patients aged $\leq 65$ compared to those aged $>65$ (median RQ: 12.954 and 10.634, respectively), but no statistically significant differences were observed ( $p>0.05$, Mann-Whitney U-test) (Table 2).

Table 2. Clinical and pathological features: median expression level (RQ value) of evaluated genes and miRNAs in tumor tissue (NSCLC).

\begin{tabular}{|c|c|c|c|c|c|c|c|c|}
\hline $\begin{array}{l}\text { Clinical and } \\
\text { Pathological } \\
\text { Features }\end{array}$ & $\begin{array}{c}C C R 7 \\
\text { (Median RQ) }\end{array}$ & $p$ Value & $\begin{array}{c}\text { CCL19 } \\
\text { (Median RQ) }\end{array}$ & $p$ Value & $\begin{array}{c}\text { miR let-7a } \\
\text { (Median RQ) }\end{array}$ & $p$ Value & $\begin{array}{c}\text { miR-335 } \\
\text { (Median RQ) }\end{array}$ & $p$ Value \\
\hline Female & 13.548 & \multirow{2}{*}{$>0.05$} & 0.853 & \multirow{2}{*}{$>0.05$} & 0.328 & \multirow{2}{*}{$>0.05$} & 3.053 & \multirow{2}{*}{$>0.05$} \\
\hline Male & 10.796 & & 1.076 & & 0.295 & & 3.554 & \\
\hline$\leq 65$ & 12.954 & \multirow[b]{2}{*}{$>0.05$} & 1.086 & \multirow[b]{2}{*}{$>0.05$} & 0.410 & \multirow[b]{2}{*}{$>0.05$} & 2.455 & \multirow{2}{*}{$>0.05$} \\
\hline$>65$ & 10.634 & & 1.052 & & 0.299 & & 3.423 & \\
\hline$<40$ PYs & 8.510 & \multirow{3}{*}{$>0.05$} & 0.712 & \multirow{3}{*}{$>0.05$} & 0.305 & \multirow{3}{*}{$>0.05$} & 3.366 & \multirow{3}{*}{$>0.05$} \\
\hline$\geq 40$ PYs & 11.299 & & 1.210 & & 0.344 & & 2.906 & \\
\hline Non-smoking & 13.548 & & 7.425 & & 0.198 & & 5.059 & \\
\hline SCC & 9.351 & \multirow{2}{*}{$>0.05$} & 1.028 & \multirow{2}{*}{$>0.05$} & 0.304 & \multirow{2}{*}{$>0.05$} & 3.292 & \multirow[b]{2}{*}{$>0.05$} \\
\hline $\mathrm{AC}$ & 13.001 & & 1.227 & & 0.315 & & 3.108 & \\
\hline AJCC & & & & & & & & \\
\hline Stage I & 11.040 & \multirow{3}{*}{$>0.05$} & 1.610 & \multirow{3}{*}{$>0.05$} & 0.250 & \multirow{3}{*}{$>0.05$} & 4.009 & \multirow{3}{*}{$>0.05$} \\
\hline Stage II & 10.504 & & 0.601 & & 0.375 & & 2.668 & \\
\hline Stage III & 11.315 & & 1.663 & & 0.260 & & 3.848 & \\
\hline pTNM (N) & & & & & & & & \\
\hline No & 12.907 & \multirow[t]{2}{*}{$>0.05$} & 1.227 & \multirow[t]{2}{*}{$>0.05$} & 0.301 & \multirow[t]{2}{*}{$>0.05$} & 3.319 & \multirow[t]{2}{*}{$>0.05$} \\
\hline $\mathrm{N} 1+\mathrm{N} 2$ & 10.504 & & 1.028 & & 0.352 & & 2.844 & \\
\hline pTNM (T) & & & & & & & & \\
\hline $\mathrm{T} 1$ & 17.897 & \multirow{3}{*}{$>0.05$} & 2.0410 & \multirow{3}{*}{$>0.05$} & 0.198 & \multirow{3}{*}{$>0.05$} & 5.059 & \multirow{3}{*}{$>0.05$} \\
\hline $\mathrm{T} 2$ & 9.351 & & 0.677 & & 0.352 & & 2.844 & \\
\hline T3 & 12.246 & & 0.899 & & 0.333 & & 3.124 & \\
\hline
\end{tabular}

The expression level of CCL19 mRNA was higher in male patients compared to female ones (median RQ: 1.076 and 0.853, respectively) but no significant differences were noted ( $p>0.05$, Mann-Whitney U-test). No statistically significant differences in CCL19 mRNA expression level were observed according to age. Slightly higher CCL19 mRNA expression level was found in those aged $\leq 65$ than $>65$ (median RQ: 1.086 and 1.052, respectively).

Regarding smoking status, higher expression levels of the mRNA were observed in patients who smoked $\geq 40$ PYs than those who smoked $<40$ PYs; however, this difference was not statistically significant ( $p>0.05$, Kruskal-Willis test) (Table 2). The greatest CCR7 and CCL19 mRNA expression level was observed between patients who smoked $\geq 40$ PYs compared to non-smokers (Table 2).

3.3. Relative Expression Levels of CCR7 and CCL19 mRNA in Tumor Tissue (NSCLC) According to Histopathological Assessment and the TNM/AJCC Staging System

Regarding histopathological type, higher CCR7 and CCL19 mRNA expression levels were observed in the AC group compared to the SCC group (Table 2); however, no statistically significant differences were observed ( $p>0.05$, Mann-Whitney U-test).

Similarly, while the greatest CCR7 and CCL19 mRNA expression levels were observed in stage III, and the lowest in stage II (Table 2), no significant correlations with cancer stage were observed according to AJCC ( $p>0.05$, Kruskal-Willis test).

Lower CCR7 and CCL19 mRNA expression levels were observed among patients with metastases to lymph nodes (N1 + N2), compared to those without them (N0). However, these differences were not statistically significant ( $p>0.05$, Mann-Whitney U-test) (Table 2). 
No significant correlations were observed between CCR7 and CCL19 mRNA expression levels and tumor sizes according to pTNM staging ( $p>0.05$, Kuskal-Wallis test). The highest CCR7 and CCL19 mRNA expressions were observed in a patient with T1 and the lowest in those with T2 (Table 2).

\subsection{Relative Expression Levels of the Study miRNAs in Tumor Tissue vs. Serum Patients with NSCLC}

All (100\%) tumor tissues demonstrated downregulated miR let-7a expression $(\mathrm{RQ}<1)$ and upregulated miR-335 expression ( $R Q>1$ ). In addition, $43 \%$ of the serum samples from patients with NSCLC demonstrated a downregulated miR-let-7a expression level and upregulated miR-335. As well, the relative expression level of miRNAs in tumor tissue was found to be significantly different to those in the serum of NSCLC patients ( $p=0.0001$, Mann-Whitney U-test) (Figure 2). A lower miR let-7a expression level was observed in tumor tissue compared to serum (median RQ: 0.309 and 1.077, respectively); however, miR-335 expression level was higher in tumor tissue than in serum (median RQ: 3.234 and 0.929 , respectively).

3.5. Relative Expression Levels of miR-335 and miR let-7a in Tumor Tissue (NSCLC) According to Biological Features and Smoking History

Regarding sex, a lower expression level of miR let-7a and higher expression level of miR-335 were observed in the group of men compared to women (Table 2); however, no significant differences were found between the study groups $(p>0.05$, Mann-Whitney U-test).

Patients aged $>65$ demonstrated a lower miR let-7a expression level and higher miR335 expression level compared to those aged 65 years and under (Table 2); however, the differences were not statistically significant ( $p>0.05$, Mann-Whitney $U$ test).
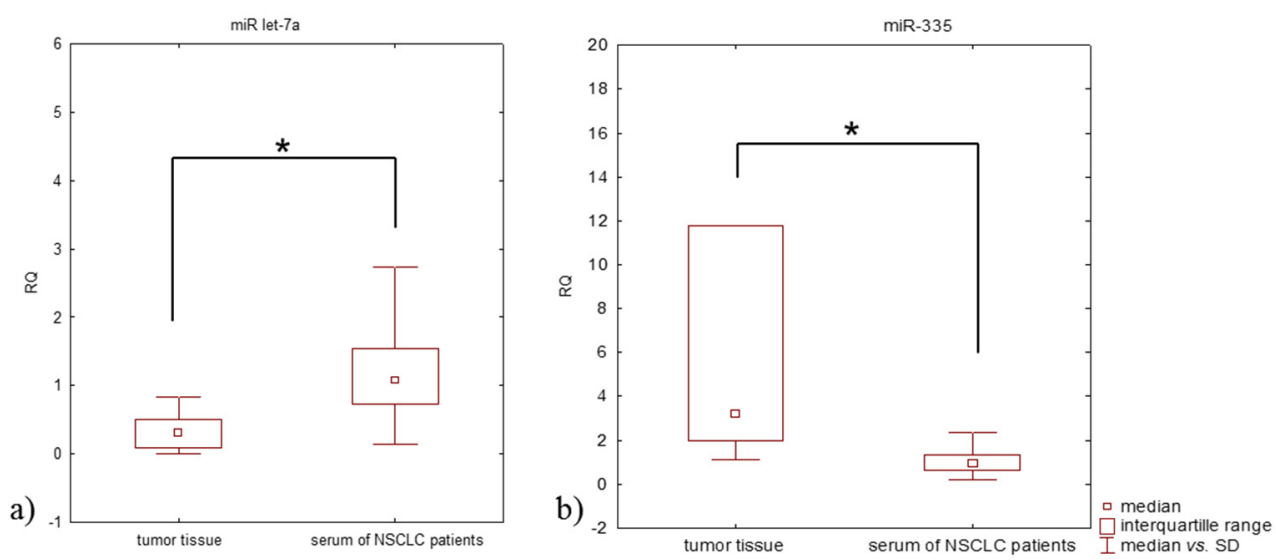

Figure 2. Box plot showing differences in median RQ values for (a) miR let-7a, (b) miR-335 in tumor tissue and in serum of NSCLC patients; ${ }^{*} p<0.05$.

Regarding smoking status, higher miR let-7a expression level was noted in patients consuming $\geq 40$ PYs compared to those consuming <40 PYs (median RQ: 0.344 and 0.305, respectively), with the lowest being observed in never-smokers (median RQ-0.198); in addition. In contrast, the highest miR-335 expression level was found in never-smokers, and the lowest in patients consuming $\geq 40$ PYs (Table 2). No statistically significant differences in the expression level of any studied miRNAs were observed with regards to smoking history ( $p>0.05$, Kruskal-Willis test)

3.6. Relative Expression Levels miR-335 and miR let-7a in Tumor Tissue (NSCLC) Classified According to Histopathological Classification and the TNM/AJCC Staging System

No statistically significant differences in miRNA expression levels were observed with regard to histopathological classification ( $p>0.05$, Mann-Whitney U-test). In SCC, the 
miRNA-335 expression level was slightly elevated, but miR let-7a expression level was decreased (Table 2).

Patients with stage I (AJCC) demonstrated the lowest miR let-7a expression level (median RQ values) and the highest miR-335 expression level (median RQ: 0.250 and 4.009, respectively). The highest miR let-7a expression level and lowest miR-335 expression level were observed in stage II (median RQ: 0.375 and 2.668, respectively). The highest miR let-7a expression was observed in stage II, followed by stage III, and then stage I. Conversely, the highest miR-335 expression was noted in stage I, followed by stage III, and then stage II. No statistically significant differences in miR-335 and miR let-7a expression levels were observed according to AJCC stage ( $p>0.05$, Kruskal-Willis test) (Table 2).

No statistically significant differences in miR-335 expression level were observed in the tissues of patients with NSCLC with regard to lymph node involvement-N (N0, $\mathrm{N} 1+\mathrm{N} 2)(p>0.05$, Mann-Whitney U-test). Lower miR-335 expression level and higher miR let-7a expression level were observed in patients with lymph node metastases (N1 + N2), compared to non-metastatic patients (N0) (Table 2).

No significant correlations were found between miR-335 expression level and tumor size (T) according to pTNM staging ( $p>0.05$, Kuskal-Wallis test). The highest miR-335 expression level was observed in a patient with $\mathrm{T} 1$ and the lowest with $\mathrm{T} 2$; this was opposite to the trend observed for miR let-7a (Table 2).

\section{7. miRNA Expression Levels in Serum from Patients with NSCLC vs. Control}

The NSCLC patients demonstrated higher miR let-7a expression level compared to controls (median RQ: 1.077 and 0.980, respectively) but lower miR-335 expression level (median RQ: 0.929 and 1.021, respectively) (Figure 3). No statistically significant differences in miR-335 and miR let-7a expression levels were observed in the study groups $(p>0.05$, Mann-Whitney U-test).
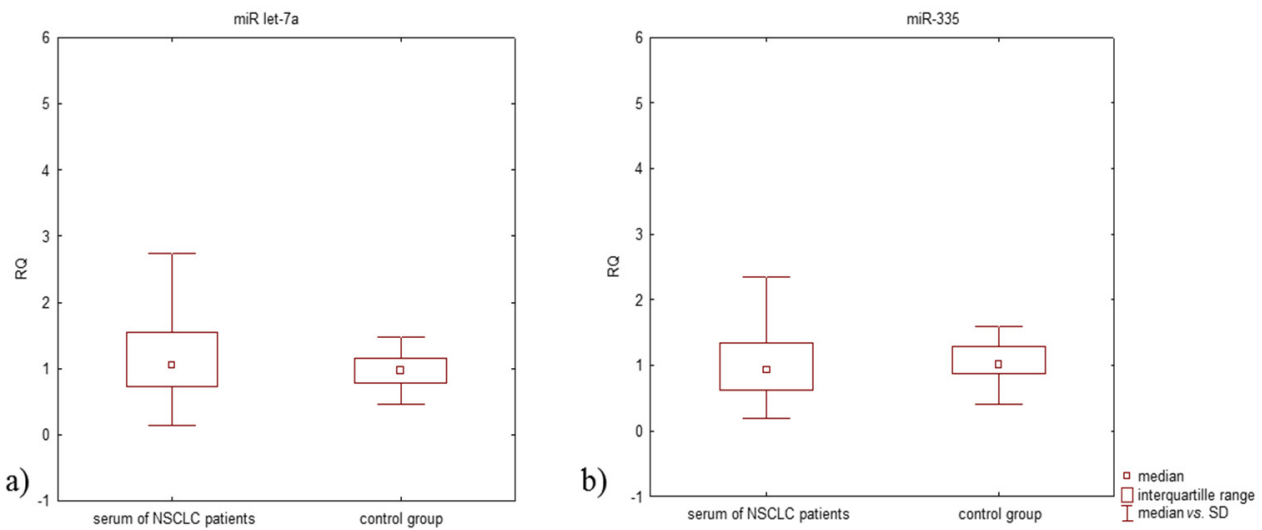

Figure 3. Box plot showing differences in median RQ values for (a) miR let-7a, (b) miR-335 in the serum of NSCLC patients and in the control group (U-Mann-Whitney test).

3.8. miR-335 and miR let-7a Expression Levels in Serum of Patients with NSCLC According to Biological Features and Smoking History

Higher miR-335 expression levels were observed among men than women (median RQ: 0.950 and 0.774 , respectively), while higher miR let-7a expression level was found among women compared to men (median RQ: 1.292 and 1.053, respectively). No significant differences in miRNA expression levels were found between the two groups $(p>0.05$, Mann-Whitney U-test).

The patients aged $\leq 65$ demonstrated lower miR let-7a expression levels but higher miR-335 expression levels than in those aged $>65$ (Table 3 ). No statistically significant differences were observed between the two groups ( $p>0.05$, Mann-Whitney U-test). 
Table 3. Clinical and pathological features: median expression level (RQ value) of evaluated miRNAs in serum (NSCLC).

\begin{tabular}{|c|c|c|c|c|}
\hline $\begin{array}{c}\text { Clinical and Pathological } \\
\text { Features }\end{array}$ & $\begin{array}{c}\text { miR let-7a } \\
\text { (Median RQ) }\end{array}$ & $p$-Value & $\begin{array}{c}\operatorname{miR}-335 \\
\text { (Median RQ) }\end{array}$ & $p$-Value \\
\hline Female & 1.292 & \multirow{2}{*}{$>0.05$} & 0.774 & \multirow{2}{*}{$>0.05$} \\
\hline Male & 1.053 & & 0.950 & \\
\hline$\leq 65$ & 0.910 & \multirow{2}{*}{$>0.05$} & 1.090 & \multirow{2}{*}{$>0.05$} \\
\hline$>65$ & 1.249 & & 0.800 & \\
\hline$<40$ PYs & 1.303 & \multirow{3}{*}{$>0.05$} & 0.768 & \multirow{3}{*}{$>0.05$} \\
\hline$\geq 40$ PYs & 0.937 & & 1.067 & \\
\hline Non-smoking & 1.292 & & 0.774 & \\
\hline SCC & 1.007 & \multirow[b]{2}{*}{0.040} & 0.993 & \multirow[b]{2}{*}{0.044} \\
\hline $\mathrm{AC}$ & 1.292 & & 0.774 & \\
\hline \multicolumn{5}{|l|}{ AJCC } \\
\hline Stage I & 1.169 & \multirow{3}{*}{$>0.05$} & 0.858 & \multirow{3}{*}{$>0.05$} \\
\hline Stage II & 1.085 & & 0.922 & \\
\hline Stage III & 0.969 & & 1.032 & \\
\hline \multicolumn{5}{|l|}{ pTNM (N) } \\
\hline No & 1.230 & \multirow[t]{2}{*}{0.026} & 0.813 & \multirow[t]{2}{*}{0.021} \\
\hline $\mathrm{N} 1+\mathrm{N} 2$ & 0.813 & & 1.230 & \\
\hline \multicolumn{5}{|l|}{ pTNM (T) } \\
\hline $\mathrm{T} 1$ & 1.107 & \multirow{3}{*}{$>0.05$} & 0.903 & \multirow{3}{*}{$>0.05$} \\
\hline $\mathrm{T} 2$ & 1.085 & & 0.922 & \\
\hline T3 & 1.030 & & 0.972 & \\
\hline
\end{tabular}

Regarding smoking status, higher miR let-7a expression was found among patients consuming $<40$ PYs than in those consuming $\geq 40$ PYs (median RQ: 1.303 and 0.937, respectively). In addition, higher miR-335 expression level was found in patients consuming $\geq 40$ PYs than in those consuming <40 PYs (median RQ: 1.067 and 0.768, respectively). No statistically significant differences were observed between the two groups $(p>0.05$, MannWhitney U-test).

3.9. miR-335 and miR let-7a Expression Levels in the Serum of Patients with NSCLC According to Histopathological Classification and the TNM/AJCC Staging System

Significant differences in miR-335 or miR let-7a expression levels were observed according to NSCLC histopathological type ( $p=0.443$ and $p=0.040$, respectively, MannWhitney U-test). Higher miRNA-335 expression was observed in SCC compared to AC (median RQ: 0.993 and 0.774, respectively). Conversely, lower miR let-7a expression level was decreased in SCC compared to AC (median RQ: 1.007 and 1.292, respectively).

Regarding the AJCC staging system, the lowest miR-335 expression level was observed in a patient with stage I (Table 3) and the highest in stage III. Conversely, the highest miR let-7a expression level was observed in stage I and the lowest in stage III. No statistically significant differences in the expression level of any studied miRNA were observed with regards to AJCC stage ( $p>0.05$, Kruskal-Willis test).

In the serum from patients with NSCLC, miR-335 expression level was found to vary significantly depending on metastasis to nearby lymph nodes $(\mathrm{N})(p=0.021$, Mann-Whitney U-test). A higher miR-335 expression level was observed in patients with lymph node metastases (N1 + N2) than in non-metastatic patients (N0) (median RQ: 1.230 and 0.813, respectively). A significantly higher miR let-7a expression level was observed in N0 vs. N1 + N2 (median RQ: 1.230 and 0.813, respectively, $p=0.026$, Mann-Whitney U-test) (Table 3).

No significant correlations were found between miR-335 expression level and primary tumor size (T) according to pTNM staging ( $p>0.05$, Kuskal-Wallis test). The highest miR335 expression was observed in a patient with T3 and the lowest in T1 (median RQ: 0.972 and 0.903 respectively). Conversely, the highest miR let-7a expression level was observed in a patient with T1 and the lowest in T3 (median RQ: 1.107 and 1.030, respectively) (Table 3). 
3.10. Correlation between the Expression Levels of the Studied Genes and miRNAs in NSCLC Patients

A significant negative correlation was found between the expression level of CCR7 and CCL19 and miR let-7a in tumor tissue $(p=0.0007$, rho $=-0.426$ and $p=0.00002$, rho $=-0.521$, respectively, Spearman's rank correlation). In addition, a significant positive correlation was observed between the expression level of CCR7 and miR-335 ( $p=0.0006$, rho $=0.430$, Spearman's rank correlation) and between the expression level of CCL19 and the currently studied miRNA $(p=0.00002$, rho $=0.524$, Spearman's rank correlation).

\section{Discussion}

Despite major advances in lung cancer treatment, no progress has been made in its early diagnosis or prediction of progression. Currently, nodal status is considered as one of the most powerful prognostic markers for resected NSCLC; therefore, improved detection techniques predicting lymph node metastasis may enable effective treatment planning for lung cancer.

Tumor cell migration shares many similarities with leukocyte trafficking, which is mainly regulated by chemokines and their receptors [2]. The CCL19/CCR7 axis plays a key role in the activation of native T cells, B cells, and dendritic cells (DCs), and the migration of these cells within lymphoid organs [19]. In NSCLC, CCR7 is expressed on the membranes of lung tumor cells, and CCR7 mRNA expression level correlates with lymph node metastasis $[2,5]$. Furthermore it was revealed that CCR7 is involved in the regulation of cell apoptosis [5], proliferation, and EMT in lung cancer cells [3].

Our study on the CCL19/CCR7 axis showed higher levels of CCR7 mRNA expression in NSCLC samples compared to macroscopically unchanged lung tissue (control tissue), surrounding the tumor. Moreover, CCR7 upregulation was observed in all study samples (tumor cells and control tissue). Our observation was consistent with previous findings [16]. Thus, in addition to the histological changes, excluding macroscopic pathological changes in the tissue surrounding the tumor, molecular and immunological changes doubtlessly also occur with tumor development. This surrounding tissue, macroscopically unchanged, being part of the molecular surgical margin (MSM), also participates in the carcinogenic process due to its heterogeneous composition and presence of cancer cells. This area has been found to demonstrate an increased rate of tumor recurrence [20]. Therefore, the surrounding tissue seems to be important in the diagnostic evaluation of NSCLC patients [21,22]. Our result (observed upregulation of the CCL19/CCR7 axis on mRNA expression level), in a macroscopically unchanged area surrounding the tumor, confirms the presence of immunological/molecular variations in this tissue.

Numerous studies highlight the value of the expression level of CCR7 mRNA and its ligand as a diagnostic molecular biomarker; however, the prognostic value has not been clearly established $[10,23]$. In the present study, the highest expression level of CCR7 mRNA was noted in patients with AJCC stage III; however, no significant differences in mRNA expression levels were found between AJCC stages I-III. Our results were similar to those of Takanaki et al. [2], who reported significant differences in CCR7 mRNA expression according to disease stage: no expression was observed for stage IA/IB (AJCC staging), and positive expression for stage III. In contrast, Liu et al. [22] reported a negative correlation between CCR7 mRNA expression levels and AJCC staging, and Itakura et al. [10] indicated that a high CCR7 mRNA expression level indicates better prognosis for AC patients and may serve as a good prognostic factor.

CCR7 is recognized as a key factor in promoting metastasis via the lymphatic system. Surpassingly, lower CCR7 mRNA expression was observed in patients with metastasis $(\mathrm{N} 1+\mathrm{N} 2)$ than in those without (N0). It should be considered whether the elevated CCR7 mRNA expression observed in AJCC stage I NSCLC patients in our study may indicate the presence of micro-metastases in the lymph nodes. However, the current staging criteria do not take into account the presence of micro-metastases in lymph nodes identified during assessments of lung cancer [23]. The increased expression of CCR7 mRNA observed in 
AJCC stage I NSCLC patients can also be understood as a "hazard signal" resulting in the activation of inflammatory mediators, leukocyte migration, or tissue damage. This hypothesis has been confirmed by other authors [24], who propose that the chemokine system is used as an antitumor immune response.

CCR7 and its ligand CCL19 control the targeting of T cells and dendritic cells to areas of the lymph nodes where the adaptive immune response is initiated. Recent studies have also shown that CCL19 has potent antitumor properties and inhibits angiogenesis [25]. In the present study, significantly higher CCL19 mRNA expression levels were observed in the tumor tissue compared to macroscopically unchanged lung tissue, which is consistent with Liu et al. [22]. In addition, the highest CCL19 mRNA expression level was noted in a case of a patient with a pTNM stage T1 primary tumor, without metastasis to lymph node (N0). It is possible that in the case of non-advanced tumors, increased CCL19 mRNA expression level may be caused by the infiltration of immune cells at the tumor site during the first stage of antitumor immunological reaction.

Itakura et al. [10] reported that high CCL19 expression level may also be a good prognostic factor, especially for AC patients. However, Liu et al. [22] note that elevated CCL19 mRNA expression level positively correlated with the presence of histologically confirmed lymph node metastasis and AJCC staging. In our present study, the highest CCL19 mRNA expression level was observed in stage III, followed by stage I, and then stage II. We therefore propose that the highest CCL19 mRNA expression may serve as a poor prognostic factor; however, this observation should be confirmed in larger groups of patients.

Smoking is considered one of the major risk factors of NSCLC development. However, little is known of the relationship between CCR7 and CCL19 mRNA expression and smoking history in NSCLC patients. Our study revealed higher CCR7 and CCL19 expression levels in patients who smoked $\geq 40$ PYs than those who smoked $<40$ PYs. It was found that smoking may correlate with elevated CCR7 mRNA expression level [26], that CCR7 plays a major role in modulating inflammatory responses in airways in pulmonary diseases [27], and that cigarette smoking upregulates CCR7, CCL19, and CCL21 mRNA expression levels in lymph nodes of wild-type mice [28]. It was also concluded that exposure to cigarette smoke can upregulate the number of DCs and cause immunoreactions through CCR7mediated chemotaxis [29]. Surpassingly, in the NSCLC patients in the present study, higher CCR7 and CCL19 expression levels were observed in non-smokers than in smokers; indeed, cigarette smoke extract (CSE) was found to suppress CCR7 expression on DCs in the lungs of smokers, but not diminish their migration towards a CCR7 ligand [28]. Our observations regarding CCR7 and CCL19 mRNA expression levels in NSCLC smokers are consistent with those of a previous study which found decreased expression of many inflammatory mediators, including several chemokines, in the BAL cells of smokers [30]. This suggests that patients with different smoking histories exhibit a different immune content and tumor immune microenvironment. Reduced CCR7 mRNA expression is an important mechanism for dendritic cell retention in the inflammatory process in the lungs of smokers during carcinogenesis.

Regarding the histopathological type of cancer, a higher CCR7 and CCL19 mRNA expression level was observed in the AC group compared to the SCC group. These results may indicate that $\mathrm{AC}$ has stronger biological aggressiveness. This is consistent with Kawase et al. [31], who reported that AC appeared to have a more aggressive nature, particularly in patients with $\mathrm{pT} 3$ or $\mathrm{pT} 4$ primary tumor size. The authors noted that patients with an AC histotype were significantly more likely to demonstrate disease recurrence.

miRNAs play a key role in the regulation of many processes underlying carcinogenesis, such as cancer cell development, differentiation, and migration [32-35]. They perform a regulatory function for numerous genes involved in metastasis, including chemokines and/or their receptors [10,11]. Moreover, miRNAs are believed to be deregulated in human cancers, emphasizing that they play an important role in tumor onset and development. 
The present study evaluates the expression level of miR-335 and miR let 7-a, which are known to be regulators of CCR7 and CCL19 mRNA expression [36,37].

$\mathrm{MiR}$ let-7a was identified as an oncosuppressor $m i R$, i.e., a tumor-suppressing miRNA [38], and miRNA-7a is known to demonstrate a different expression profile based on the tumor type. Jeong et al. [39] reported lower miR let-7a expression levels in tumor NSCLC tissue compared to control tissue. Our present findings indicate miR let-7a downregulation $(\mathrm{RQ}<1)$ in all NSCLC tissue samples, and that miR let-7a expression level was downregulated $(\mathrm{RQ}<1)$ in $43 \%$ of serum NSCLC patient samples. Interestingly, miRNA-7a expression was significantly reduced in tissue compared to serum. Our observation is consistent with those of Jeong et al. [39] regarding NSCLC patients. This suggests that miR let-7a plays a significant role in the local tumor specific regulation of targeted genes involved in lung carcinogenesis. It should be pointed that miR let-7a affects many genes which play a key role in cell pathophysiology, including carcinogenesis. Downregulation of miR let-7a in lung cancer cells is associated with greater self-sufficiency in growth signals, insensitivity to antigrowth signals, limitless replicative potential, and the ability to avoid immune surveillance [34]. However, the full mechanism of miR let-7a regulation in NSCLC remains unclear. Research suggests that miR let-7a may regulate proliferation, apoptosis, and invasion in lung cell lines [38,40,41].

Of course, the statistically significant differences in the miR let-7a expression level between the tissue samples and serum exosomes observed in our study should not be surprising. Indeed, miRNA expression is tissue-specific, as confirmed in previous studies based on unrelated human tissues and their exosomal origin [42-44].

Our findings indicate a negative correlation between the expression level of miR let-7a obtained from serum exosomes and the stage of cancer development according to the AJCC and pTNM classifications. Significantly lower expression levels of the studied miRNAs were observed in the serum of a patient with lymph node metastasis, and this correlation was not observed in tumor tissue. The expression level of miR let-7a in the serum of NSCLC patients was previously found to correlate with disease progression, i.e., the stage of cancer and metastasis to lymph nodes [16]. Our data are consistent with that obtained in a meta-analysis performed by Pop-Bica et al. [45], who found miR let-7a to be downregulated in different sample types (tissue, FFPE tissue, serum, serum/plasma, or exosomes) and to predict a poor outcome in NSCLC patients. This data, and those of our present findings, indicate that miR let-7a expression level may serve as a prognostic biomarker in the liquid biopsy of these NSCLC patients.

To identify non-invasive diagnostic markers, the present study compared the expression of miR let-7a in sera from NSCLC patients and healthy controls. No statistically significant differences were found in the miR let-7a expression level between the study groups; however, higher expression was observed in the NSCLC patients. In contrast, Ying et al. [46] reported significantly lower expression levels of miR let-7a in serum from NSCLC patients compared with healthy controls, and noted that the panel for miRNAs including miR let-7a is able to detect NSCLC development in the early stage of disease. Some differences exist between our present findings and those of other studies; however, these may be due to a range of factors including patient infection, peripheral inflammation, smoking status, epigenetic modification of miR let-7a, or differences in patient numbers.

miRNA expression has been found to play a significant role in smoking-mediated oncogenic events in cancer and their diagnostic/prognostic potential $[34,47,48]$. It is wellknown that smoking is considered a carcinogenesis risk factor, especially in the lung [49]. Therefore, our present study examines the relationship between the miR let-7a expression level in tumor tissue and the number of cigarettes smoked (<40 PYs vs. $>40$ PYs). It is commonly known that most miRNAs demonstrate varying expression through all carcinogenetic stages, from the normal bronchi of non-smokers to invasive SCC in smokers. Most miRNAs were initially downregulated, in agreement with the hypothesis that miRNA downregulation often occurs in cancer where tissue is losing its normal differentiation. A positive correlation was observed in the present study between the miR let-7a expres- 
sion level in tumor tissue samples and smoking status. The highest expression level was observed among patients who smoked more than 40 PYs. However, a lower expression level of miR let-7a was observed in the serum of patients consuming $\geq 40 \mathrm{PYs}$ than those consuming < 40 PYs. These results are consistent with those obtained by Rizk et al. [49], who observed a significant negative correlation between $\mathrm{miR}$ let-7a expression level and pack years in the serum of smokers.

The present study also focuses on miR-335 expression level in NSCLC patents, which is involved in the regulation of epithelial-mesenchymal transition (EMT), proliferation, and migration of lung cancer cells [50]. Huo et al. [51] indicated than miR-335 still plays a suppressive role in NSCLC tumorigenesis, and that the downregulation of miR-335 in lung cancer cells promoted cell proliferation through upregulation of Tra2 $\beta$ [52]. Significantly lower miR-335 expression levels were also found in NSCLC tissue samples compared to control lung tissue. In addition, in the present study, the miR-335 expression level was upregulated in all tumor tissues and in $43 \%$ of the serum of NSCLC patients. These findings have been confirmed by Scalora et al. [53], who noted an increased expression level of miR-335 in lung cancer cells and confirmed its association with a higher proliferation rate and progression. Unfortunately, our studies did not identify any association between miR355 expression dysregulation and cancer progression. Our analysis did not reveal any correlation between the miR-335 expression level in tumor tissue and stage of cancer development according to AJCC and pTNM classifications. In contrast, Huo et al. [51] found that miR-335 expression level in tumor tissue significantly correlated with lymph node metastasis and pathological TNM stage.

Moreover, our findings do not confirm any relationship between the miR-335 expression level in tumor tissue samples/serum and the age or sex of study patients. These observations are consistent with those of other researchers $[51,54]$.

Interestingly, a negative correlation was identified between the miR-335 expression level in tumor samples and smoking history: a lower miR-335 expression level was observed in smokers compared to non-smokers, and decreased as smoking intensity increased (PYs $<40$ vs. PYs $>40$ ). Ong et al. [55] reported that current smokers demonstrated a lower miR-335 expression level in primary lung fibroblasts compared to ex-smokers. Downregulated miR-335 expression may enhance fibroblast proliferation by targeting $\mathrm{Rb} 1$, CARF, and SGK3.

Lower miR-335 expression levels were observed in the serum of NSCLC patients compared to controls; however, the differences were not statistically significant. This is the first such report of miR-355 deregulation; however, decreased miR-335 expression level has been noted in the serum of patients with various malignant tumors, such as hepatocellular carcinoma (HCC) [56], gastric cancer [57], and gallbladder cancer (GC) [58]. Ciu et al. [56] reported that HCC patients with lower miR-335 expression levels in serum had significantly poorer prognosis than those with higher serum expression. This observation suggests that miR-335 may have prognostic value. Moreover, Wu et al. [58] noted a negative correlation between the miR-335 expression level and lymph node metastasis in GC patients. Our present findings indicate high miR-335 expression level in serum from NSCLC patients, and that this level correlated with cancer progression based on clinical tumor stage (AJCC), lymph node involvement and tumor size. Hence, miR-335 expression level may serve as a biomarker with negative prognostic value in the liquid biopsy of NSCLC patients. Similarly, Sun et al. [59] indicated that exosomal miRNA-335 enhances invasion and metastasis in colorectal cancer by facilitating EMT via targeting RASA1.

Our present findings indicate that CCR7/CCL19 mRNA expression level has a significant negative correlation with $\mathrm{miR}$ let-7a expression level and a positive correlation with miR-335. Similar findings regarding the direct regulatory influence of CCR7/CCL19 mRNA and miR let-7a have been obtained in experiments with Luciferase reporter assays [60]. It has also been documented that $\mathrm{miR}$ let-7a directly regulates CCR7 protein expression through interaction with $3^{\prime} \mathrm{UTR}$ of CCR7. 
The limitation of our research was that only small groups of patients were included in the study. This may also be the reason why relatively few statistically significant results were obtained regarding the relationship between the expression level of CCR7/CCL19 mRNA, study miRNAs, and cancer features. Moreover, according to the recommended NSCLC treatment algorithm and indication for surgical treatment (depending on the advancement of the lesion), it was difficult for us to obtain an equal number of tissue samples for study groups (e.g., I-III according to TNM/AJCC staging).

\section{Conclusions}

Changes in the level of CCL19/CCR7 mRNA expression in tumor tissue can be considered as a diagnostic marker for NSCLC patients. The CCR7 and its ligand CCL19, play two important but challenging roles in NSCLC. On the one hand, the CCR7/CCL19 axis has a key role in promoting metastasis via the lymphatic system. On the other hand, the CCL19/CCR7 axis is involved in the modulation of the immune response in a growing tumor. It should be noted that the role of CCL19/CCR7 in NSCLC appears to be complex, and post-transcriptional regulation of these genes may be important in the progression and metastasis of this cancer.

Significant differences in miR let-7a and miR-335 expression levels were found between the tumor tissue and serum of NSCLC patients, indicating that miR let-7a has a suppressive function in the primary lesion and that miR-335 has an oncogenic one. Our findings confirm previous observations indicating miR-335 and miR let-7a expression levels in the serum of patients with NSCLC correlate with lymph node metastasis. The studied miRNAs may potentially serve as non-invasive molecular biomarkers with prognostic value (prediction of metastatic potential) in liquid biopsy.

Author Contributions: Study conception and design, E.B.-L. and K.B.; material preparation and data collection, J.K., S.J. and K.B.; data analysis, K.B.; first draft of the manuscript, K.B.; revised manuscript E.B.-L. and A.A. All authors commented on subsequent versions of the manuscript. All authors have read and agreed to the published version of the manuscript.

Funding: This study was funded by the Medical University of Lodz (Statute No. 503/1-013-02/50311-003 and the National Centre for Research and Development (Warsaw, Poland) within the grant InterChemMed (WND-POWR.03.02.00-00-I029/16-01).

Institutional Review Board Statement: The study was approved by the Bioethics Committee of the Medical University of Lodz, Poland (resolution No. KE/149/18).

Informed Consent Statement: Informed consent was obtained from all subjects involved in the study.

Conflicts of Interest: The authors declare no conflict of interest.

\section{References}

1. Cancer Today. Available online: http://gco.iarc.fr/today/home (accessed on 25 August 2021).

2. Takanami, I. Overexpression of CCR7 mRNA in nonsmall cell lung cancer: Correlation with lymph node metastasis. Int. J. Cancer 2003, 105, 186-189. [CrossRef] [PubMed]

3. Zhang, L.; Xiao, X.; An, H.; Wang, J.; Ma, Y.; Qian, Y.H. Inhibition of CCR7 promotes NF-kB-dependent apoptosis and suppresses epithelial-mesenchymal transition in non-small cell lung cancer. Oncol. Rep. 2017, 37, 2913-2919. [CrossRef] [PubMed]

4. Sun, L.; Zhang, Q.; Li, Y.; Tang, N.; Qiu, X. CCL21/CCR7 up-regulate vascular endothelial growth factor-D expression via ERK pathway in human non-small cell lung cancer cells. Int. J. Clin. Exp. Pathol. 2015, 8, 15729-15738.

5. Xu, Y.; Liu, L.; Qiu, X.; Liu, Z.; Li, H.; Li, Z.; Luo, W.; Wang, E. CCL21/CCR7 prevents apoptosis via the ERK pathway in human non-small cell lung cancer cells. PLoS ONE 2012, 7, e33262. [CrossRef] [PubMed]

6. Xu, Y.; Liu, L.; Qiu, X.; Jiang, L.; Huang, B.; Li, H.; Li, Z.; Luo, W.; Wang, E. CCL21/CCR7 Promotes G2/M Phase Progression via the ERK Pathway in Human Non-Small Cell Lung Cancer Cells. PLoS ONE 2011, 6, e21119. [CrossRef]

7. Pu, J.; Tang, X.; Zhuang, X.; Hu, Z.; He, K.; Wu, Y.; Dai, T. Matrine induces apoptosis via targeting CCR7 and enhances the effect of anticancer drugs in non-small cell lung cancer in vitro. Innate Immun. 2018, 24, 394-399. [CrossRef]

8. Cardell, M.; Arni, S.; Yang, S.C.; Korom, S.; Opitz, I.; Lardinois, D.; Sharma, S.; Dubinett, S.M.; Weder, W.; Hillinger, S. Combined CCL19/IL-7 treatment eradicates tumors in murine models of lung cancer. Cancer Res. 2006, 66, 1306. 
9. Cheng, H.W.; Onder, L.; Cupovic, J.; Boesch, M.; Novkovic, M.; Pikor, N.; Tarantino, I.; Rodriguez, R.; Schneider, T.; Jochum, W.; et al. CCL19-producing fibroblastic stromal cells restrain lung carcinoma growth by promoting local antitumor T-cell responses. J. Allergy Clin. Immunol. 2018, 142, 1257-1271.e4. [CrossRef]

10. Itakura, M.; Terashima, Y.; Shingyoji, M.E.; Yokoi, S.; Ohira, M.; Kageyama, H.; Matui, Y.; Yoshida, Y.; Ashinuma, H.; Moriya, Y.; et al. High CC chemokine receptor 7 expression improves postoperative prognosis of lung adenocarcinoma patients. Br. J. Cancer 2013, 109, 1100-1108. [CrossRef]

11. Lu, Y.; Luan, X.R. miR-147a suppresses the metastasis of non-small-cell lung cancer by targeting CCL5. J. Int. Med. Res. 2020, 48, 300060519883098. [CrossRef]

12. Li, J.; Tang, Z.; Wang, H.; Wu, W.; Zhou, F.; Ke, H.; Lu, W.; Zhang, S.; Zhang, Y.; Yang, S.; et al. CXCL6 promotes non-small cell lung cancer cell survival and metastasis via down-regulation of miR-515-5p. Biomed. Pharmacother. 2018, 97, 1182-1188. [CrossRef] [PubMed]

13. Mirzaei, S.; Zarrabi, A.; Hashemi, F.; Zabolian, A.; Saleki, H.; Ranjbar, A.; Saleh, S.H.; Bagherian, M.; omid Sharifzadeh, S.; Hushmandi, K.; et al. Regulation of Nuclear Factor-KappaB (NF-кB) signaling pathway by non-coding RNAs in cancer: Inhibiting or promoting carcinogenesis? Cancer Lett. 2021, 509, 63-80. [CrossRef] [PubMed]

14. Ge, Q.; Zhou, Y.; Lu, J.; Bai, Y.; Xie, X.; Lu, Z. miRNA in Plasma Exosome is Stable under Different Storage Conditions. Molecules 2014, 19, 1568-1575. [CrossRef] [PubMed]

15. Peng, Y.; Croce, C.M. The role of MicroRNAs in human cancer. Signal Transduct. Target. Ther. 2016, 1, 15004. [CrossRef]

16. Baran, K.; Kiszałkiewicz, J.; Migdalska-Sęk, M.; Jabłoński, S.; Kordiak, J.; Antczak, A.; Góralska, K.; Brzeziańska-Lasota, E. An assessment of the relationship between the expression of CCR7/CCL19 axis and selected regulatory miRNAs in non-small cell lung cancer. Mol. Biol. Rep. 2019, 46, 5389-5396. [CrossRef]

17. Available online: https://www.cancer.gov/publications/dictionaries/cancer-terms/def/pack-year (accessed on 20 August 2021).

18. Mirsadraee, S.; Oswal, D.; Alizadeh, Y.; Caulo, A.; van Beek, E.J. The 7th lung cancer TNM classification and staging system: Review of the changes and implications. World J. Radiol. 2012, 4, 128-134. [CrossRef]

19. Comerford, I.; Harata-Lee, Y.; Bunting, M.D.; Gregor, C.; Kara, E.E.; McColl, S.R. A myriad of functions and complex regulation of the CCR7/CCL19/CCL21 chemokine axis in the adaptive immune system. Cytokine Growth Factor Rev. 2013, 24, 269-283. [CrossRef]

20. Mao, L.; Clark, D. Molecular margin of surgical resections-Where do we go from here? Cancer 2015, 121, 1914-1916. [CrossRef]

21. Kalinowska-Herok, M.; Roś, M.; Widłak, P. Tumor molecular margins. Nowotw. J. Oncol. 2013, 63, 28-34.

22. Liu, Y.; Wu, B.Q.; Geng, H.; Xu, M.L.; Zhong, H.H. Association of chemokine and chemokine receptor expression with the invasion and metastasis of lung carcinoma. Oncol. Lett. 2015, 10, 1315-1322. [CrossRef]

23. Carretta, A. Clinical value of nodal micrometastases in patients with non-small cell lung cancer: Time for reconsideration? J. Thorac. Dis. 2016, 8, E1755-E1758. [CrossRef] [PubMed]

24. Labani-Motlagh, A.; Ashja-Mahdavi, M.; Loskog, A. The Tumor Microenvironment: A Milieu Hindering and Obstructing Antitumor Immune Responses. Front. Immunol. 2020, 11, 940. [CrossRef] [PubMed]

25. Hillinger, S.; Yang, S.C.; Batra, R.K.; Strieter, R.M.; Weder, W.; Dubinett, S.M.; Sharma, S. CCL19 reduces tumour burden in a model of advanced lung cancer. Br. J. Cancer 2006, 94, 1029-1034. [CrossRef] [PubMed]

26. Liao, S.X.; Ding, T.; Rao, X.M.; Sun, D.S.; Sun, P.P.; Wang, Y.J.; Fu, D.D.; Liu, X.L.; Ou-Yang, Y. Cigarette smoke affects dendritic cell maturation in the small airways of patients with chronic obstructive pulmonary disease. Mol. Med. Rep. 2015, 11, 219-225. [CrossRef]

27. Demoor, T.; Bracke, K.R.; Vermaelen, K.Y.; Dupont, L.; Joos, G.F.; Brusselle, G.G. CCR7 modulates pulmonary and lymph node inflammatory responses in cigarette smoke-exposed mice. J. Immunol. 2009, 183, 8186-8194. [CrossRef] [PubMed]

28. Vassallo, R.; Walters, P.R.; Lamont, J.; Kottom, T.J.; Eunhee, S.Y.; Limper, A.H. Cigarette smoke promotes dendritic cell accumulation in COPD; a Lung Tissue Research Consortium study. Respir. Res. 2010, 11, 45. [CrossRef] [PubMed]

29. Zhang, J.F.; Li, Y.; Zhang, A.Z.; He, Q.Q.; Du, Y.C.; Cao, W. Expression and pathological significance of CC chemokine receptor 7 and its ligands in the airway of asthmatic rats exposed to cigarette smoke. J. Thorac. Dis. 2018, 10, 5459. [CrossRef]

30. Meuronen, A.; Majuri, M.L.; Alenius, H.; Mäntylä, T.; Wolff, H.; Piirilä, P.; Laitinen, A. Decreased cytokine and chemokine mRNA expression in bronchoalveolar lavage in asymptomatic smoking subjects. Respiration 2008, 75, 450-458. [CrossRef]

31. Kawase, A.; Yoshida, J.; Ishii, G.; Nakao, M.; Aokage, K.; Hishida, T.; Nishimura, M.; Nagai, K. Differences between squamous cell carcinoma and adenocarcinoma of the lung: Are adenocarcinoma and squamous cell carcinoma prognostically equal? Jpn. J. Clin. Oncol. 2012, 42, 189-195. [CrossRef]

32. Wang, M.; Yu, F.; Ding, H.; Wang, Y.; Li, P.; Wang, K. Emerging Function and Clinical Values of Exosomal MicroRNAs in Cancer. Mol. Ther. Nucleic Acids 2019, 16, 791-804. [CrossRef]

33. Iqbal, M.A.; Arora, S.; Prakasam, G.; Calin, G.A.; Syed, M.A. MicroRNA in lung cancer: Role, mechanisms, pathways and therapeutic relevance. Mol. Aspects Med. 2019, 70, 3-20. [CrossRef] [PubMed]

34. Momi, N.; Kaur, S.; Rachagani, S.; Ganti, A.K.; Batra, S.K. Smoking and microRNA dysregulation: A cancerous combination Trends Mol. Med. 2014, 20, 36-47. [CrossRef] [PubMed]

35. Iorio, M.V.; Croce, C.M. MicroRNAs in cancer: Small molecules with a huge impact. J. Clin. Oncol. 2009, 27, 5848-5856. [CrossRef] [PubMed] 
36. Available online: https://mirtarbase.cuhk.edu.cn/ \{\}miRTarBase/miRTarBase_2019/php/search.php?org=hsa\&opt=mirna_ id\&kw=mir-335 (accessed on 20 August 2021).

37. Available online: https://mirtarbase.cuhk.edu.cn/ \{\}miRTarBase/miRTarBase_2019/php/search.php?org=hsa\&opt=mirna_ id\&kw $=$ hsa-let-7a (accessed on 20 August 2021).

38. Zhao, W.; Hu, J.X.; Hao, R.M.; Zhang, Q.; Guo, J.Q.; Li, Y.J.; Xie, N.; Liu, L.Y.; Wang, P.Y.; Zhang, C.; et al. Induction of microRNA-let-7a inhibits lung adenocarcinoma cell growth by regulating cyclin D1. Oncol. Rep. 2018, 40, 1843-1854. [CrossRef] [PubMed]

39. Jeong, H.C.; Kim, E.K.; Lee, J.H.; Yoo, H.N.; Kim, J.K. Aberrant expression of let-7a miRNA in the blood of non-small cell lung cancer patients. Mol. Med. Rep. 2011, 4, 383-387. [CrossRef] [PubMed]

40. Wang, Y.Y.; Ren, T.; Cai, Y.Y.; He, X.Y. MicroRNA let-7a inhibits the proliferation and invasion of nonsmall cell lung cancer cell line 95D by regulating K-Ras and HMGA2 gene expression. Cancer Biother. Radiopharm. 2013, 28, 131-137. [CrossRef]

41. Duan, S.; Yu, S.; Yuan, T.; Yao, S.; Zhang, L. Exogenous Let-7a-5p Induces A549 Lung Cancer Cell Death Through BCL2L1Mediated PI3K $\gamma$ Signaling Pathway. Front. Oncol. 2019, 9, 808. [CrossRef]

42. Ludwig, N.; Leidinger, P.; Becker, K.; Backes, C.; Fehlmann, T.; Pallasch, C.; Rheinheimer, S.; Meder, B.; Stähler, C.; Meese, E.; et al. Distribution of miRNA expression across human tissues. Nucleic Acids Res. 2016, 44, 3865-3877. [CrossRef]

43. Zhang, J.; Li, S.; Li, L.; Li, M.; Guo, C.; Yao, J.; Mi, S. Exosome and Exosomal MicroRNA: Trafficking, Sorting, and Function. Genom. Proteom. Bioinform. 2015, 13, 17-24. [CrossRef]

44. Guduric-Fuchs, J.; O'Connor, A.; Camp, B.; O’Neill, C.L.; Medina, R.J.; Simpson, D.A. Simpson, Selective extracellular vesiclemediated export of an overlapping set of microRNAs from multiple cell types. BMC Genom. 2012, 13, 357. [CrossRef]

45. Pop-Bica, C.; Pintea, S.; Magdo, L.; Cojocneanu, R.; Gulei, D.; Ferracin, M.; Berindan-Neagoe, I. The Clinical Utility of miR-21 and let-7 in Non-small Cell Lung Cancer (NSCLC). A Systematic Review and Meta-Analysis. Front. Oncol. 2020, 10, 2210. [CrossRef] [PubMed]

46. Ying, L.; Du, L.; Zou, R.; Shi, L.; Zhang, N.; Jin, J.; Xu, C.; Zhang, F.; Zhu, C.; Wu, J.; et al. Development of a serum miRNA panel for detection of early stage non-small cell lung cancer. Proc. Natl. Acad. Sci. USA 2020, 117, 25036-25042. [CrossRef] [PubMed]

47. Mathis, C.; Poussin, C.; Weisensee, D.; Gebel, S.; Hengstermann, A.; Sewer, A.; Belcastro, V.; Xiang, Y.; Ansari, S.; Wagner, S.; et al. Human bronchial epithelial cells exposed in vitro to cigarette smoke at the air-liquid interface resemble bronchial epithelium from human smokers. Am. J. Physiol. Lung Cell. Mol. Physiol. 2013, 304, L489-L503. [CrossRef]

48. Huang, W.; Li, M.D. Differential allelic expression of dopamine D1 receptor gene (DRD1) is modulated by microRNA miR-504. Biol. Psychiatry 2009, 65, 702-705. [CrossRef]

49. Malhotra, J.; Malvezzi, M.; Negri, E.; La Vecchia, C.; Boffetta, P. Risk factors for lung cancer worldwide. Eur. Respir. J. 2016, 48, 889-902. [CrossRef]

50. Du, W.; Tang, H.; Lei, Z.; Zhu, J.; Zeng, Y.; Liu, Z.; Huang, J.A. miR-335-5p inhibits TGF- $\beta 1$-induced epithelial-mesenchymal transition in non-small cell lung cancer via ROCK1. Respir. Res. 2019, 20, 225. [CrossRef]

51. Huo, W.; Zhang, M.; Li, C.; Wang, X.; Zhang, X.; Yang, X.; Fei, H. Correlation of microRNA-335 expression level with clinical significance and prognosis in non-small cell lung cancer. Medicine 2020, 99, e21369. [CrossRef]

52. Liu, J.; Bian, T.; Feng, J.; Qian, L.; Zhang, J.; Jiang, D.; Zhang, Q.; Li, X.; Liu, Y.; Shi, J. miR-335 inhibited cell proliferation of lung cancer cells by target Tra2 $\beta$. Cancer Sci. 2018, 109, 289-296. [CrossRef] [PubMed]

53. Scarola, M.; Schoeftner, S.; Schneider, C.; Benetti, R. miR-335 directly targets Rb1 (pRb/p105) in a proximal connection to p53-dependent stress response. Cancer Res. 2010, 70, 6925-6933. [CrossRef]

54. Tang, H.; Zhu, J.; Du, W.; Liu, S.; Zeng, Y.; Ding, Z.; Zhang, Y.; Wang, X.; Liu, Z.; Huang, J. CPNE1 is a target of miR-335-5p and plays an important role in the pathogenesis of non-small cell lung cancer. J. Exp. Clin. Cancer Res. 2018, 37, 131. [CrossRef]

55. Ong, J.; van den Berg, A.; Faiz, A.; Boudewijn, I.M.; Timens, W.; Vermeulen, C.J.; Oliver, B.G.; Kok, K.; Terpstra, M.M.; van den Berge, M.; et al. Current Smoking is Associated with Decreased Expression of miR-335-5p in Parenchymal Lung Fibroblasts. Int. J. Mol. Sci. 2019, 20, 5176. [CrossRef]

56. Cui, L.; Hu, Y.; Bai, B.; Zhang, S. Serum miR-335 Level is Associated with the Treatment Response to Trans-Arterial Chemoembolization and Prognosis in Patients with Hepatocellular Carcinoma. Cell Physiol. Biochem. 2015, 37, 276-283. [CrossRef]

57. Sandoval-Bórquez, A.; Polakovicova, I.; Carrasco-Véliz, N.; Lobos-González, L.; Riquelme, I.; Carrasco-Avino, G.; Bizama, C.; Norero, E.; Owen, G.I.; Roa, J.C.; et al. MicroRNA-335-5p is a potential suppressor of metastasis and invasion in gastric cancer. Clin. Epigenet. 2017, 9, 114. [CrossRef] [PubMed]

58. Wu, T.; Li, Y.; Huang, C.; Li, X. Serum levels and significances of miR-335 and miR-155 in primary gallbladder cancer. J. Int. Oncol. 2019, 46, 267-271.

59. Sun, X.; Lin, F.; Sun, W.; Zhu, W.; Fang, D.; Luo, L.; Li, S.; Zhang, W.; Jiang, L. Exosome-transmitted miRNA-335-5p promotes colorectal cancer invasion and metastasis by facilitating EMT via targeting RASA1. Mol. Ther.-Nucleic Acids 2021, 24, 164-174. [CrossRef] [PubMed]

60. Kim, S.J.; Shin, J.Y.; Lee, K.D.; Bae, Y.K.; Sung, K.W.; Nam, S.J.; Chun, K.H. MicroRNA let-7a suppresses breast cancer cell migration and invasion through downregulation of C-C chemokine receptor type 7. Breast Cancer Res. 2012, 14, R14. [CrossRef] [PubMed] 